\title{
MEASURING BEHAVIOR 2000 Editorial
}

The articles in the August 2001 issue of Behavior Research Methods, Instruments, \& Computers are based on presentations that were made at the 3rd International Conference on Methods and Techniques in Behavioral Research, which was held in Nijmegen, The Netherlands, on August 15-18, 2000. The program chair and conference host was Professor Alexander Cools, Department of Psychoneuropharmacology, University of Nijmegen.

Measuring Behavior is a biennial conference on methods and techniques in behavioral research. It is an attempt to create bridges between disciplines by bringing people together who might otherwise be unlikely to meet. At a Measuring Behavior meeting, one finds ethologists; behavioral ecologists; behavioral neuroscientists; developmental, social, and work psychologists; psychiatrists; linguists; psychophysiologists; toxicologists; ergonomists, human factors researchers, movement scientists, and usability testers. Although the research questions and applications may be highly diverse, all delegates share an interest in methods, techniques, and tools for the study of human or animal behavior. The focus on methodological and technical themes can lead to a very productive crossfertilization between research fields.

Measuring Behavior 2000 brought together more than 300 delegates from 35 countries. During 3 conference days, there were 165 presentations grouped in 13 thematic symposia. Besides reading papers, delegates presented posters and demonstrated software or equipment. The program also included scientific tours, technical training sessions, special interest groups, workshops, and user meetings organized by vendors, as well as an exhibition of scientific books, instruments, and software.

The articles published in this issue of BRMIC illustrate the variety of technical topics addressed at a Measuring Behavior meeting. Of course, they represent only a small selection of the presentations that were actually made. The proceedings may be found at http://www.noldus.com/events/mb2000/. Printed copies of the proceedings can be obtained from the Conference Secretariat, P. O. Box 268, 6700 AG Wageningen, The Netherlands (fax: +31-317-424496, e-mail: mb2000@ noldus.nl).

Sixteen authors have provided articles for the Measuring Behavior issue of BRMIC. The first five papers address the elemental behavioral units of human communication: Bente et al. describe the use of 3-D animation techniques for the study of nonverbal interaction; Neidle et al. present a system for the transcription and analysis of gestural language; Nathani and Oller analyze strategies for coding infants' prelinguistic vocalizations; Yoder et al. analyze the sequential association of events in dyadic interactions; and Thomann proposes a content-free metric for identifying important events in interaction.

Turning to movements on a larger scale, Bussmann et al. use accelerometry to characterize overt behavior during daily activities, and Tulen et al. use a similar technique for the quantification of motor tics in Tourette's syndrome.

Several authors share methods of characterizing animal behavior with the use of video-based methods: Šustr et al. evaluate interactions among pigs; Mukhina et al. automatically analyze rats' performance in the Morris water maze; Twining et al. recognize postures in rats; Ohl et al. evaluate rats' responses to novel environments; and Noldus et al. describe the commercial EthoVision video tracking system that many animal behavior researchers employ. 
Using behavioral methods, Zarcone and Fowler exploit disk pressing force as a dependent measure of rat behavior; Clarke et al. measure ground reaction forces in the mouse's locomotion; Cooper and Mason observe behavioral allocation in mink; and Rowan et al. compare human and rodent sequential response learning.

The 4th International Conference Conference of Methods and Techniques in Behavioral Research, Measuring Behavior 2002, will be held in Amsterdam, The Netherlands, on August 27-30, 2002. Details can be found on the conference web site, http://www.noldus.com/events/mb2002/, or e-mail inquiries may be directed to mb2002@noldus.nl.

Jonathan Vaughan Hamilton College 